\title{
Community paramedicine: A patch, or a real system improvement?
}

\author{
Peter Cameron, MBBS, $\mathrm{MD}^{*}$; Alix Carter ${ }^{(1)}, \mathrm{MD}, \mathrm{MPH}^{\ddagger \S}$
}

\section{INTRODUCTION}

Community paramedicine is a program that uses paramedics to provide immediate or scheduled primary, urgent, and/or specialized healthcare to vulnerable patient populations by focusing on improving equity in healthcare access across the continuum of care, as defined by the Canadian Standards Association, Paramedic Chiefs of Canada, and Paramedic Association of Canada in the national standard called Community paramedicine: Framework for program development. ${ }^{1}$ In addition to Canadian leadership, there is considerable effort being made internationally to develop community paramedicine. The International Roundtable on Community Paramedicine (www.ircp.info) and the Rural and Frontier EMS Agenda for the Future, published by the US Department of Health and Human Services, are international leaders. Examples of these programs can be found through the above organizations as well as the American College of Emergency Physicians (www. acep.org), http://www.communityparamedic.ca, and the Paramedic Chiefs of Canada (www.emscc.ca). Mobile Integrated Healthcare Practice is a term that is sometimes used to describe similar programs. ${ }^{2}$ As programs develop and evolve, and there are comparisons and calls for standardization, it is important to fully recognize the scope of community paramedicine programs beyond just scheduled, home-based care; the Canadian Standards Association standard and national paramedic leadership has a broader definition of community paramedicine that includes alternative responses to 9-1-1 calls, provision of care using existing skills in a differentiated manner, and coordination of care (Figure 1). ${ }^{1}$

A variety of factors have contributed to the emergence of community paramedicine. Paramedicine has become a health profession and has been key in advancing community paramedicine. There are also political pressures at play, with fewer community physician resources and increasing community expectations of $24 / 7$ medicine. There are local issues such as poorly serviced urban areas, residential aged care, under insurance, and drug and alcohol programs. As such, community paramedicine programs have been developed one of two ways: Paramedicine has seen a problem and filled a gap, or (less desirable perhaps) it has come from political pressure. Because of these various factors, programs risk being a patch instead of a well-thought-out system of improvement. Ideally, programs are well-thought-out and based on needs assessment, standards, and (inter)national collaboration.

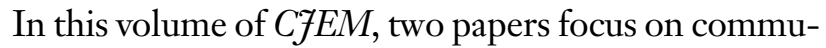
nity paramedicine. Chan et al. present a systematic review describing training programs for community paramedicine as published in the peer reviewed literature. ${ }^{3}$ Leyenaar et al. present an environmental scan of the style/format/type of assessment conducted by homevisit type community paramedicine programs in Ontario, Canada. ${ }^{4}$ Both conclude that better sharing (publication) of community paramedicine program information and more standardization would improve quality and equity across programs.

From the *Emergency and Trauma Centre, The Alfred Hospital, Melbourne, Australia; †Health Services Division, School Public Health and Preventive Medicine, Monash University, Melbourne, Australia; ¥Division of Emergency Medical Services (EMS), Dalhousie University Department of Emergency Medicine; and the §Research, Emergency Health Services (EHS), Halifax, NS.

Correspondence to: Dr. Alix Carter, Dalhousie University Department of Emergency Medicine, Divison of EMS, 3021-1796 Summer St., Halifax, NS B3H 3A7, Canada; Email: Alix.carter@ novascotia.ca

(C) Canadian Association of Emergency Physicians

CJEM 2019;21(6):691-693

DOI 10.1017/cem.2019.439 


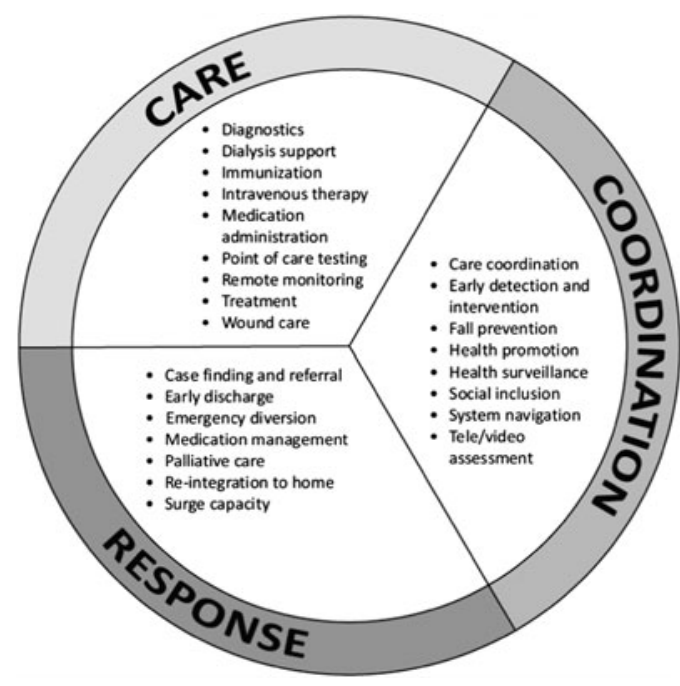

Figure 1. Different models of community paramedicine.

\section{DIRECTION FOR THE FUTURE}

The emergence of these varied and diverse services under the umbrella "Community paramedicine" begs the question: What does the health system really need so that we evolve services that will strengthen the system and improve population health in a sustainable way? Of course, a complex problem such as this rarely has a single magic bullet, but what role can and should community paramedicine play in evolving a sustainable and effective healthcare system?

One of the risks of rooting a program like this in the foundation of paramedicine is that, as emergency clinicians, we look at it through an acute care lens. We have reactive responses, repeatedly pulling people out of the river instead of figuring out why they are falling in. Wisely spent healthcare resources can build more resilient and healthy populations through community-based care, and well planned health systems anticipate the need for unscheduled care without requiring an emergency response. This is not to say that the emergency response system has no role to play in healthy communities, but to say that there are conditions better managed in a planned (albeit at times unscheduled) manner; wellmanaged ambulatory care sensitive conditions have fewer crises. ${ }^{5,6}$ It is essential that we ensure we aren't just putting more resources into stop-gap, piece-meal solutions. The risk of stop-gap programs is that they could in fact channel patients into acute care rather than chronic, preventive, community-based care. Successful community paramedicine programs need to include non-transport and alternative destinations as outcomes of 9-1-1 calls, intentional communication online and offline between emergency medical services (EMS) and community care providers, and design of programs with all of those players at the table to strengthen the overall function of an integrated health system. The current inexorable annual increase in ambulance transports across advanced healthcare systems is not sustainable.

Through the lens of major problems facing the health system are as follows:

- Chronic diseases - diabetes/frailty/falls, and so forth. These are Ambulatory Care Sensitive Conditions; when well managed, emergency response requirements decrease. ${ }^{5,6}$ Though the lack of primary care physicians is a limitation, other novel solutions may exist through an enhanced and integrated primary care infrastructure, better suited to deliver preventive services. Examples, including community paramedicine, exist. ${ }^{7}$ Careful thought and planning should be given to how to best use all available resources.

- Elderly residential care facilities. Novel approaches exist to better designing long-term care programs to mitigate the development of these crises or novel ways to respond to crises when they arise, such as the Care by Design program, which integrates dedicated primary care with non-transport responses by community paramedics. ${ }^{8}$ Again, careful thought and planning is key and should be cross-sectoral and intentional.

- Rural areas. This is an issue underrepresented in the accompanying papers but critical to the sustainability of healthcare. What is the role for enhanced skill sets for clinic nurses and/or with telehealth support? Should rural paramedics provide scheduled care, too? Can alternative responses to 9-1-1 leverage the training and infrastructure of the EMS system as an effective/efficient way to reach the population, enhancing primary care instead of channeling into acute care? Examples of home care partnerships and wellness clinics have increased rural access to care. ${ }^{9}$

Community paramedicine can be an important part of evolving our healthcare system. Paramedics have the unique training and experience of walking into chaotic unknown situations and bringing calm. Paramedic training includes important and unique perspectives, such as a scene size-up, a rapid primary and secondary survey, what does the home look like, how is the family coping, 
also a different experience with (some) independence of action - that is, follow a protocol without interacting with a physician or getting an order. Paramedics also have access to various medications, and a response model using existing ground ambulance coverage designed to reach $90 \%$ of calls in a fairly short time frame, which is one of the critical elements for patients, and may not efficiently be addressed by a system in which a dedicated individual responds to community paramedicine requests over a large territory with low demand. ${ }^{10}$ The system integration approach should consider appropriately using the skills of community paramedics and integrating with and supporting other providers as appropriate.

These articles both conclude by articulating the need for standards. This is a key step in moving a young program forward. However, standards can be detrimental if done poorly, failing to recognize unique local needs or resources. Standards can't be so rigid or narrow that they miss the richness of local needs assessment and innovation, and also the richness of what may be happening outside of the local geography or model of community paramedicine. High-level standards like the Canadian Standards Association are helpful, in that they describe stakeholder engagement, appropriate education beyond current paramedic foundational training, sustainability, and a quality assurance program.

In summary, community paramedicine holds promise to be an important part of the evolving healthcare landscape. It is critical that these programs, as with all healthcare reform, be evolved in an integrated inter-sectoral collaboration. Piece-meal patches to a system in crisis can run the risk of inequities, difficulty in doing benchmarking or research, and unintended redirection away from a broken primary care system. The importance of preventive healthcare in the management of ambulatory care sensitive conditions cannot be forgotten. Efforts like those put forth by Leyenaar ${ }^{4}$ and $\mathrm{Chan}^{3}$ to call for information sharing and collaboration to advance the science and practice of community paramedicine will be important as these programs continue to emerge.
Keywords: Prehospital / EMS, community paramedicine, health system planning

Competing interests: None declared.

\section{REFERENCES}

1. Standards Council of Canada. Community paramedicine standard; 2018. Available at: https://www.csagroup.org/article/ cancsa-z1630/ (accessed November 11, 2018).

2. Mobile Integrated Healthcare.com. Community paramedicine has evolved in response to a health system crisis/revolution; 2013. Available at: http://www.mobileintegratedhealthcare. com/Websites/mihc/images/MIHP_whitepaper_FINAL.pdf (accessed November 11, 2018).

3. Chan J, Griffith L, Costa A, Leyenaar M, Agarwal G. Community paramedicine: a systematic review of program descriptions and training. CFEM 2019;21(6):749-61.

4. Leyenaar M, Mcleod B, Penhearow S, et al. What do community paramedics assess? An environmental scan and content analysis of patient assessment in community paramedicine. CFEM 2019;21(6):766-75.

5. Purdy S, Griffin T, Salisbury C, Sharp D. Ambulatory care sensitive conditions: terminology and disease coding need to be more specific to aid policy makers and clinicians. Public Health 2009;123(2):169-73.

6. Caminal J, Starfield B, Sanchez E, Casanova C, Morales M. The role of primary care in preventing ambulatory care sensitive conditions. Eur 7 Public Health 2004;14(3):246-51.

7. Agarwal G, Angeles R, Pirrie M, McLeod B, Marzanek F. Evaluation of a community paramedicine health promotion and lifestyle risk assessment program for older adults who live in social housing: a cluster randomized trial. CMA7 2018;190:E638-47.

8. Marshall EG, Clarke B, Peddle S, Jensen JL. Care by Design: a new model of care in long-term care facilities providing residents coordinated on-site primary and acute care. Can Fam Physician 2015;61:e129-34.

9. Saskatoon Health Region. Available at: https://www.saska toonhealthregion.ca/locations_services/Services/Pre-HospitalEmergency/Pages/Community-Paramedicine-.aspx (accessed November 11, 2018).

10. Carter AJE, Arab M, Harrison M, et al. Paramedics providing palliative care at home: a mixed-methods exploration of patient and family satisfaction and paramedic comfort and confidence. CFEM 2019;21(4):513-22. 